\title{
Desarrollo de yogur tipo III con zapallo y ajonjolí como aporte de fibra y antioxidantes
}

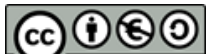

Developmente of yogurt type III with pumkin and ajonjoli as a contribution of fiber and antioxidants Title

Anabel de los Ángeles Morales Caluña. ${ }^{1}$, Fredy Patricio Erazo Rodríguez. ${ }^{2}$, Armando Vinicio Paredes Peralta. ${ }^{3} \&$ Tatiana Elizabeth Sánchez Herrera. ${ }^{4}$

\begin{abstract}
.
DOI: https://doi.org/10.33262/concienciadigital.v3i2.1.1222

The research was carried out at the Food Production Plan of the School of Animal Sciences of the ESPOCH, a type III yogurt with pumpkin and three different levels $(3,5$ and $7 \%)$ of sesame seeds was developed, compared to a control treatment $(0 \%$ sesame),distributed under a completely randomized design, with four repetitions per treatment and an experimental unit size of one liter of yogurt, were subjected to the following statistical analyzes: Analysis of Variance (ADEVA), separation of means by Tukey $(\mathrm{P}<0.05)$, regression and correlation analysis and sensory analysis with the affective hedonic scale. Determining that the physical chemical properties were affected statistically presenting a $\mathrm{pH}$ of 4.32 and 4.47 for 0 and $7 \%$ sesame levels respectively; The dry matter content of 19.35 and $23.93 \%$ ( 0 and $7 \%$ of sesame seeds), Protein of 2.60 and 3.30 ( 0 and $5 \%$ of sesame seeds), Fat of 0.54 and 3.18 ( 0 and $7 \%$

\footnotetext{
${ }^{1}$ Empresa ALAMBA, Riobamba, Ecuador, amorales0314@gmail.com

${ }^{2}$ Escuela Superior Politécnica de Chimborazo, Facultad de Ciencias Pecuarias. Riobamba, Ecuador. fredy.erazo@ espoch.edu.ec

3 Escuela Superior Politécnica de Chimborazo, Facultad de Ciencias Pecuarias. Riobamba, Ecuador. armando.paredes@espoch.edu.ec

4 Escuela Superior Politécnica de Chimborazo, Facultad de Ciencias Pecuarias. Riobamba, Ecuador. tatiana.sanchez@espoch.edu.ec
} 
of sesame seeds), Antioxidant capacity of $0.53 \%$ and $1.24 \%$ correspond to level 0 and $7 \%$ of sesame seeds. Microbiological analyzes determined the absence of pathogenic microorganisms. In relation to organoleptic characteristics, these were statistically influenced, yogurt made with $5 \%$ sesame seeds had the highest score of 4/5, which gives a rating of likes in terms of color, smell, taste and texture characteristics; therefore it is recommended to use $5 \%$ sesame seeds in the manufacture of yogurt.

Keywords: Yogurt; pumpkin; sesame seed; microbiological analysis; organoleptical analysis.

\section{Resumen.}

La presente investigación se llevó a cabo en la Planta de Producción de Alimentos de la Facultad de Ciencias Pecuarias de la ESPOCH, se desarrolló un yogur tipo III con zapallo y tres diferentes niveles $(3,5$ y $7 \%$ ) de ajonjolí, frente a un tratamiento control (0\% de ajonjolí), distribuidas bajo un diseño completamente al azar, con cuatro repeticiones por tratamiento y un tamaño de unidad experimental de un litro de yogur, fueron sometidas a los siguientes análisis estadísticos: Análisis de Varianza (ADEVA), separación de medias por Tukey $(\mathrm{P}<0,05)$, análisis de la regresión y correlación y análisis sensorial con la escala hedónica afectiva. Determinándose que las propiedades físico químicas se vieron afectadas estadísticamente presentando un $\mathrm{pH}$ de 4.32 y 4.47 para 0 y $7 \%$ niveles de ajonjolí respectivamente; el contenido de materia seca de 19.35 y $23.93 \%$ ( 0 y $7 \%$ de ajonjolí), Proteína de 2.60 y 3.30 (0 y 5\% de ajonjolí), Grasa de 0.54 y 3.18 ( 0 y $7 \%$ de ajonjolí), Capacidad antioxidante de $0.53 \%$ y $1.24 \%$ corresponde al nivel 0 y $7 \%$ de ajonjolí. Los análisis microbiológicos determinaron la ausencia de microorganismos patógenos. En relación a las características organolépticas estas se vieron influenciadas estadísticamente, el yogur elaborado con el 5\% de ajonjolí presentó el mayor puntaje de 4/5 que da una calificación de me gusta en cuento a características de color, olor, sabor y textura; por lo tanto se recomienda utilizar el 5\% de ajonjolí en la elaboración de yogur.

Palabras claves: Yogurt; zapallo; semillas de ajonjolí; análisis microbiológico; análisis organoléptico.

\section{Introducción.}

En nuestro país existen alimentos naturales que tienen componentes nutricionales como el zapallo, rico en contenido de fibra, indispensables para llevar una vida saludable, además el ajonjolí es una gran fuente de antioxidantes.

La utilización de zapallo y ajonjolí, para la elaboración de yogur tipo III es de suma importancia, para ser una alternativa en el campo de los yogures con aporte de fibra y antioxidantes, de esta manera generar un producto nutricional que ayude en la salud de los consumidores. (Bareiro, 2011: p.12). 
Por esta razón se ve la necesidad de evaluar el aporte de fibra del zapallo y antioxidantes del ajonjolí en el yogur tipo III, aplicando bases de tecnología de lácteos, con el propósito de mejorar la funcionalidad del producto. Esta característica va ligada con el sabor, la textura, el color y el olor del producto, de igual manera es función de la calidad de los ingredientes y de su mezcla base.

El valor nutritivo del yogur se considera que está relacionado con la leche que se utiliza, por cuanto el yogur contiene más proteínas, tiamina y riboflavina que la leche, pero menos vitamina A, hay poca diferencia entre el contenido de los elementos nutritivos que suministran energía de la leche y los del yogur. (Porter, 1981: pág. 12).

Por lo que se planteó desarrollar yogur tipo con zapallo y ajonjolí como aporte de fibra y antioxidantes.

\section{Metodologia.}

La presente investigación se realizó en el Laboratorio de Procesamiento de Alimentos de la Facultad de Ciencias Pecuarias de la ESPOCH, que está ubicado en el cantón Riobamba Kilómetro 1 1 1/2 Panamericana sur. La cual tuvo un tiempo de duración estimado de 120 días.

Se evaluó la adición de tres concentraciones de ajonjolí (3,5 y $7 \%$ ) en la elaboración de yogur tipo III con mermelada de zapallo al $40 \%$ de concentración, frente a un tratamiento control (0\% de ajonjolí), por lo que las unidades experimentales fueron distribuidas bajo un Diseño Completamente al Azar (DCA), con 4 repeticiones por tratamiento, dando un total de 16 unidades experimentales

Se realizaron Análisis Fisicoquímicos para determinación de $\mathrm{pH}$, materia seca, proteína, grasa, fibra y capacidad antioxidante; Análisis Microbiológicos para control de Coliformes totales, Staphylococcus aureus, E coli y Mohos y levaduras y Análisis Sensoriales para valorar parámetros de color, olor, sabor y textura.

\section{Metodología de Evaluación}

\section{Análisis fisicoquímicos}

pH: Se realizó con el Método de NTE INEN 389.

Si la muestra es líquida, homogeneizarla convenientemente mediante agitación.

Si la muestra corresponde a productos densos o heterogéneos, homogeneizarla con ayuda de una pequeña cantidad de agua (recientemente hervida y enfriada) con agitación.

Materia Seca: Método de Desecación en Estufa de Aire Caliente 
Pesar 1-10g de muestra (previamente realizado su demuestre) en vidrio reloj, pesa filtro o en papel aluminio o chocolatín: o directamente en la cápsula de porcelana previamente tarada, repetir uniformemente en su base.

Colocar en la estufa a $103{ }^{\circ} \mathrm{C} \pm 3{ }^{\circ} \mathrm{C}$ por un lapso de 2 a 3 horas, hasta peso constante. Enfriar en desecador hasta temperatura ambiente y pesar.

Proteína: Con el Método de Microkjeldhal, pesar exactamente 40mg de muestra seca e introducirla en el balón de digestión Kjeldhal. Añadir: 1,5g de K2SO4 o NaSO4; 40mg de $\mathrm{HgO}, 2 \mathrm{~mL}$ de ácido sulfúrico concentrado p.a procurando no manchar las paredes del mismo. Colocar el balón en el digestor y calentar hasta obtener un líquido transparente.

Enfriar el balón y su contenido, adicionar $4 \mathrm{~mL}$ de agua destilada para disolver el contenido que al enfriarse se solidifica.

Grasa: Mediante el Método de Gerber en donde se lleva la muestra a una temperatura de aproximadamente $20^{\circ} \mathrm{C}$, y mezclarla mediante agitación suave hasta que este homogénea, cuidando que no haya separación de grasa por efecto de la agitación.

Verter 10mL exactamente medidos, de ácido sulfúrico en el butirómetro respectivo, cuidando de no humedecer con ácido el cuello del butirómetro.

Fibra: Por el Método de Weende se debe pesar $2 \mathrm{~g}$ de muestra seca y desengrasada y colocar en el vaso de Berzellius con núcleos de ebullición y $250 \mathrm{~mL}$ de ácido sulfúrico 1,25\%. Colocar el vaso en el equipo y ajustar al condensador, subir la parrilla y calentar hasta ebullición. Mantener la ebullición por media hora exacta, contados partir de que empieza a hervir. Desconectar el vaso del condensador, enfriar y filtrar al vacío.

Capacidad antioxidante: A través de Cromatografía de capa fina para determinar Vitamina E Principalmente, pueden utilizarse dos modos de cromatografía (fase normal y fase reversa) para la cuantificación de los tocoferoles. El sistema de fase normal tiene claras ventajas dado que todos los vitámeros son separados mientras que los sistemas de fase reversa no separan $\beta$-tocoferol de y-tocoferol.

\section{Análisis organolépticos}

Se emplearon 15 panelistas (no capacitados) en la evaluación sensorial. Se efectuaron 4 repeticiones del panel en 2 diferentes días. Se repartió $40 \mathrm{ml}$ por tratamiento a cada panelista y se repartieron las muestras de yogur por cada tratamiento y agua para neutralizar el paladar. Las características sensoriales evaluadas fueron las siguientes: color, olor, sabor y textura. Se evaluaron los 4 tratamientos con una escala hedónica de 5 puntos. 


\section{Análisis microbiológicos}

Recuento de Coliformes totales: Simultáneamente con el ensayo confirmatorio de la Norma INEN 1 529-6 inocular dos o tres asas de cada uno de los tubos presuntamente positivos en un tubo conteniendo $10 \mathrm{ml}$ de caldo BGBL y en otro que contenga aproximadamente $3 \mathrm{ml}$ de caldo triptona. Incubar estos tubos a $45,5 \pm 0,2^{\circ} \mathrm{C}$ (baño María) por 48 horas y hacer el recuento.

Recuento de Staphylococcus aureus: En placa de siembra por extensión en superficie. Preparar con anticipación las placas de agar Baird Parker y secar su superficie. Preparar el homogeneizado y las diluciones del alimento. Inocular placas duplicadas, con $0,1 \mathrm{ml}$ de las diluciones escogidas. -Extender los inóculos con el extensor de vidrio, flameando cada vez luego de su uso. Dejar abiertas las placas frente al mechero, durante 15 minutos hasta que se sequen. Incubar en posición invertida a $37^{\circ} \pm 1^{\circ} \mathrm{C}$. Durante $36 \pm 2$ horas.

\section{Recuento de mohos y levaduras.}

En placa por siembra en profundidad utilizando una sola pipeta estéril, pipetear, por duplicado, alícuotas de $1 \mathrm{ml}$ de cada una de las diluciones decimales en placas Petri adecuadamente identificadas.

Iniciar por la dilución de menor concentración. Inmediatamente, verter en cada una de las placas inoculadas, aproximadamente $20 \mathrm{ml}$ de agar sal-levadura de Davis (SLD) fundido y templado a $45 \pm 2{ }^{\circ} \mathrm{C}$. La adición del medio de cultivo no debe pasar más de 15 minutos, a partir de la preparación de la primera dilución.

Los resultados experimentales fueron modelados utilizando un Diseño Completamente al Azar simple utilizando el programa estadístico INFOSTAT (versión 2017) y los análisis estadísticos fueron:

Análisis de varianza (ADEVA), Separación de medias, mediante la prueba de Tukey al 0,05 de significancia. Y la Prueba sensorial se realizó una estadística no paramétrica para variables organolépticas.

\section{Resultados}

Análisis de resultados fisicoquímicos del yogur tipo III con zapallo y ajonjolí como aporte de fibra y antioxidantes.

En la Tabla 1, se puede ver los resultados de los parámetros fisicoquímicos del yogur tipo III con zapallo y ajonjolí a los días 1,10 y 21 . 
ISSN: 2600-5859

Tabla 1. Características fisicoquímicas del yogur tipo III con zapallo y ajonjolí como aporte de fibra y antioxidantes.

\begin{tabular}{|c|c|c|c|c|c|c|c|c|c|c|}
\hline \multirow{3}{*}{\begin{tabular}{l} 
Variable \\
\multicolumn{1}{c}{ pH } \\
Día 1
\end{tabular}} & \multicolumn{8}{|c|}{ Tratamientos } & \multirow{3}{*}{$\frac{\text { E.E. }}{\text { O.O1 }}$} & \multirow{3}{*}{$\begin{array}{c}\text { Prob. } \\
\text { OO.OOO }\end{array}$} \\
\hline & \multicolumn{2}{|c|}{$0 \%$} & \multicolumn{2}{|c|}{$3 \%$} & \multicolumn{2}{|c|}{$5 \%$} & \multicolumn{2}{|c|}{$7 \%$} & & \\
\hline & 4.37 & $\mathbf{a}$ & 4.45 & B & 4.47 & $\mathbf{b}$ & 4.54 & $\mathrm{c}$ & & \\
\hline Día 10 & 4.30 & $\mathbf{a}$ & 4.35 & $\mathbf{B}$ & 4.43 & $\mathbf{b}$ & 4.46 & c & 4,2OE-O2 & $<0.0001$ \\
\hline Día 21 & 4.29 & $\mathbf{a}$ & 4.32 & $\mathbf{B}$ & 4.38 & c & 4.41 & d & O.O1 & $<0.0001$ \\
\hline Variable & \multicolumn{8}{|c|}{ Tratamientos } & \multirow{2}{*}{ E.E. } & \multirow{2}{*}{ Prob. } \\
\hline Materia seca & \multicolumn{2}{|c|}{$0 \%$} & \multicolumn{2}{|c|}{$3 \%$} & \multicolumn{2}{|c|}{$5 \%$} & \multicolumn{2}{|c|}{$7 \%$} & & \\
\hline Día 10 & 19.36 & $\mathbf{a}$ & 20.75 & $\mathbf{b}$ & 23.44 & c & 23.97 & d & 0.06 & $<0.0001$ \\
\hline Día 21 & 19.40 & $\mathbf{a}$ & 20.72 & $\mathbf{b}$ & 23.45 & c & 23.84 & c & 0.15 & $<0.0001$ \\
\hline Variable & \multicolumn{8}{|c|}{ Tratamientos } & \multirow{2}{*}{ E.E. } & \multirow{2}{*}{ Prob. } \\
\hline Proteína & \multicolumn{2}{|c|}{$0 \%$} & \multicolumn{2}{|c|}{$3 \%$} & \multicolumn{2}{|c|}{$5 \%$} & \multicolumn{2}{|c|}{$7 \%$} & & \\
\hline Día 1 & 2.61 & $\mathbf{a}$ & 3.04 & $\mathbf{b}$ & 3.35 & $\mathrm{C}$ & 3.26 & d & 0.02 & $<0.0001$ \\
\hline Día 10 & 2.61 & $\mathbf{a}$ & 3.03 & $\mathbf{b}$ & 3.29 & C & 3.29 & c & O.O1 & $<0.0001$ \\
\hline Día 21 & 2.58 & $\mathbf{a}$ & 3.04 & $\mathbf{b}$ & 3.28 & C & 3.30 & c & 0.02 & $<0.0001$ \\
\hline Grasa & \multicolumn{2}{|c|}{$0 \%$} & \multicolumn{2}{|c|}{$3 \%$} & \multicolumn{2}{|c|}{$5 \%$} & \multicolumn{2}{|c|}{$7 \%$} & E.E. & Prob. \\
\hline Día 1 & 0.57 & $\mathbf{a}$ & 1.29 & $\mathbf{b}$ & 2.15 & $\mathrm{c}$ & 3.15 & d & O.O1 & $<0.0001$ \\
\hline Día 10 & 0.53 & $\mathbf{a}$ & 1.34 & $\mathbf{b}$ & 2.18 & c & 3.18 & c & 0.03 & $<0.0001$ \\
\hline Día 21 & 0.54 & $\mathbf{a}$ & 1.30 & $\mathbf{b}$ & 2.17 & c & 3.21 & d & 0.02 & $<0.0001$ \\
\hline Variable & & & & ata & entos & & & & $\pi$ & 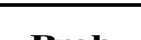 \\
\hline Fibra & & & & & & & 7 & & E. & TFOB. \\
\hline Día 1 & 2.25 & $\mathbf{a}$ & 2.54 & B & 2.64 & $\mathrm{c}$ & 2.89 & d & 0.02 & $<0.0001$ \\
\hline Día 10 & 2.25 & $\mathbf{a}$ & 2.53 & $\mathbf{B}$ & 2.65 & $\mathbf{b}$ & 2.90 & d & 0.02 & $<0.0001$ \\
\hline Día 21 & 2.26 & $\mathbf{a}$ & 2.56 & B & 2.64 & b & 2.87 & c & 0.02 & $<0.0001$ \\
\hline Variable & & & & ats & entos & & & & 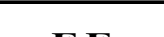 & D. \\
\hline Vitamina E & & & & & & & 7 & & E.E. & Prob. \\
\hline Día 1 & nd & $\mathbf{a}$ & 0.55 & $\mathbf{b}$ & 0.84 & $\mathrm{c}$ & 1.24 & d & O.O1 & $<0.0001$ \\
\hline Día 10 & nd & $\mathbf{a}$ & 0.52 & $\mathbf{b}$ & 0.83 & c & 1.27 & d & O.O1 & $<0.0001$ \\
\hline
\end{tabular}

Fuente: Elaboración propia.

\section{E.E $=$ Error Estándar}

Prob > 0,05: no existe diferencias estadísticas

Prob < 0,05: existe diferencias significativas

Prob $<0,01$ : existen diferencias altamente significativas.

Medias con letras iguales en la misma fila no difieren estadísticamente de acuerdo a la prueba de Tukey.

\section{pH.}

Los valores medios determinados para el $\mathrm{pH}$ del yogur tipo III reportaron diferencias altamente significativas en los días 1,10 y 21 ( $\mathrm{P}<0.0001)$, por efecto de los distintos niveles de ajonjolí, presentando el valor más alto el tratamiento con $7 \%$ de ajonjolí en el día 1, mientras que el valor más bajo fue en el tratamiento con $0 \%$ de ajonjolí al día 21.

El análisis de la regresión se muestra en el Gráfico 1, en donde se registró una respuesta de segundo orden (cuadrática) con la siguiente ecuación y $=-0.0156 \times 2+0.1712 x+3.9419$ y un 
coeficiente de determinación de 93\%; que nos expresa que el pH depende de los niveles de ajonjolí en un $93 \%$.

Gráfico 1. Regresión del contenido de $\mathrm{pH}$ en el yogur elaborado con distintos niveles de ajonjolí día 21.

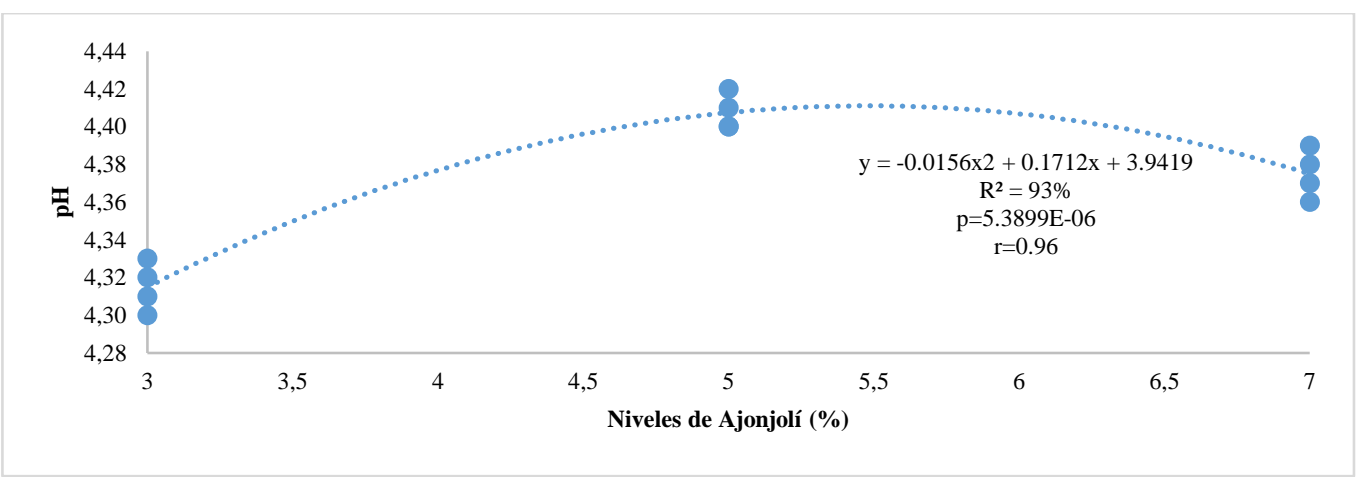

Fuente: Elaboración propia.

\section{Materia seca}

Los valores medios determinados para la materia seca del yogur tipo III reportaron diferencias altamente significativas en los días 1,10 y 21 ( $\mathrm{P}<0.0001)$, por efecto de los distintos niveles de ajonjolí, presentando el valor más alto el tratamiento con $7 \%$ de ajonjolí en el día 1, 10 y 21; mientras que el valor más bajo fue en el tratamiento con $0 \%$ de ajonjolí al día 1,10 y 21.

El análisis de regresión se muestra en el Gráfico 2 para el contenido de materia seca de yogur en el día 21 en donde se registró una respuesta de segundo orden (cuadrática) ya que por cada nivel de ajonjolí que se incluya en el yogur la materia seca se incrementa en 3.69 unidades hasta el 6,5\% de nivel de ajonjolí añadido y luego disminuye en 0.29 unidades; con un coeficiente de determinación $\mathrm{R}=95 \%$.

Gráfico 2. Regresión del contenido de materia seca en el yogur elaborado con distintos niveles de ajonjolí día 21

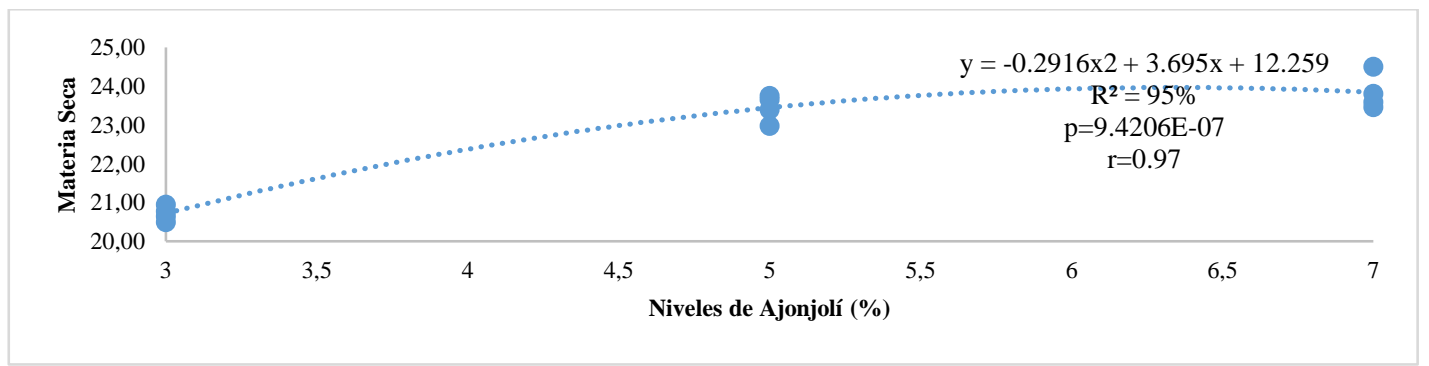

Fuente: Elaboración propia. 


\section{Proteína}

Los valores medios determinados para la proteína del yogur tipo III reportaron diferencias altamente significativas en los días 1,20 y 21 ( $\mathrm{P}<0.0001)$, por efecto de los distintos niveles de ajonjolí, presentando el valor más alto el tratamiento con 5\% de ajonjolí en el día 1, mientras que el valor más bajo fue en el tratamiento con $0 \%$ de ajonjolí al día 21 .

El análisis de la regresión se muestra en el Gráfico 3, en donde se registró una respuesta de segundo orden (cuadrática) con la siguiente ecuación y $=-0.0341 \mathrm{x} 2+0.3987 \mathrm{x}+2.1528$ y un coeficiente de determinación de $91 \%$; que nos expresa que la proteína depende de los niveles de ajonjolí en un $91 \%$.

Gráfico 3. Regresión del contenido de proteína en el yogur elaborado con distintos niveles de ajonjolí día 21

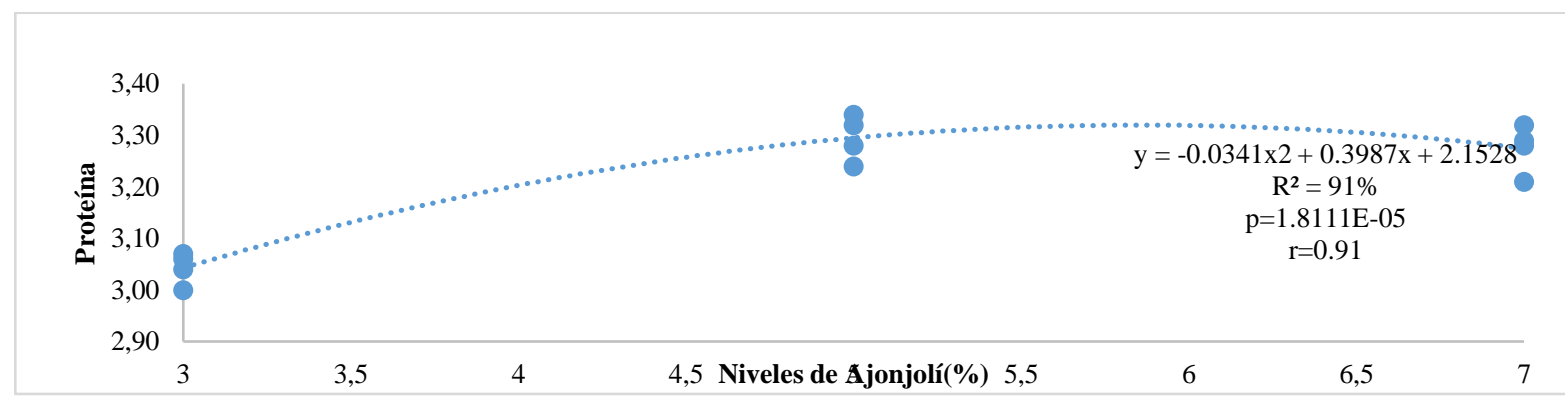

Fuente: Elaboración propia.

\section{Grasa}

Las medias del contenido de grasa o extracto etéreo de los yogures elaborados con las diferentes niveles de ajonjoli, presentaron diferencias estadísticas altamente significativas entre tratamientos $(\mathrm{P}>0.0001)$, por cuanto al emplearse $0 \%$ de ajonjolí presentó un contenido de grasa de $0.57,0.53$ y $0.54 \%$ en los días 1,10 y 21 respectivamente; mientras que al incorporar niveles de ajonjolí el contenido de grasa se incrementan cabe recalcar que se está incorporando grasa vegetal propia del ajonjolí, lo que hace referencia que el yogur es tipo III por la grasa de la leche.

El análisis de la regresión se muestra en el Gráfico 4, en donde se registró una respuesta de primer orden (lineal) con la siguiente ecuación $\mathrm{y}=0,021 \times 2+0,261 \mathrm{x}+0,322$ y un coeficiente de determinación de $99 \%$; que nos expresa que la grasa depende de los niveles de ajonjolí en un $99 \%$. 
Gráfico 4. Regresión del contenido de grasa en el yogur elaborado con distintos niveles de ajonjolí día 21.

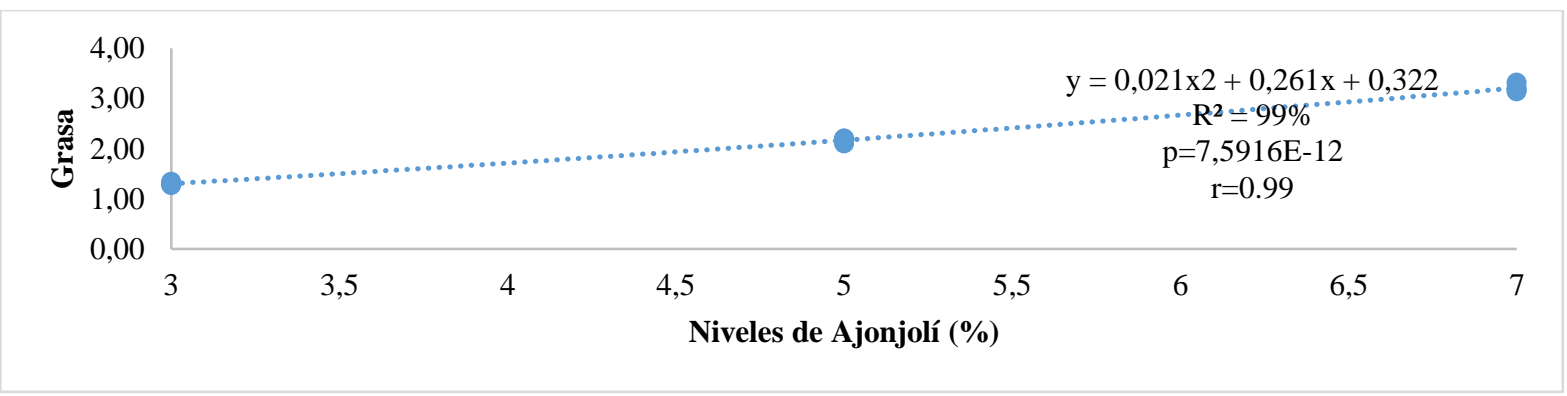

Fuente: Elaboración propia.

\section{Fibra.}

El porcentaje de fibra en los tratamientos difieren estadísticamente $(\mathrm{P}<0.001)$. El valor promedio más alto es $2.88 \%$ corresponde al nivel $7 \%$ de ajonjolí, los niveles 0,3 y $5 \%$ contienen un porcentaje promedio de $2.25,2.54$ y $2.64 \%$ de fibra.

El análisis de regresión se muestra en el Gráfico 5 para el contenido de fibra en el yogur al día 21 en donde se registró una respuesta de primer orden (lineal) con la siguiente ecuación $\mathrm{y}=0.0781 \mathrm{x}+2.2994 \mathrm{y}$ un coeficiente de determinación de $82 \%$; que nos expresa que la fibra depende de los niveles de ajonjolí en un $82 \%$.

Gráfico 5. Regresión del contenido de fibra en el yogur elaborado con distintos niveles de ajonjolí día 21.

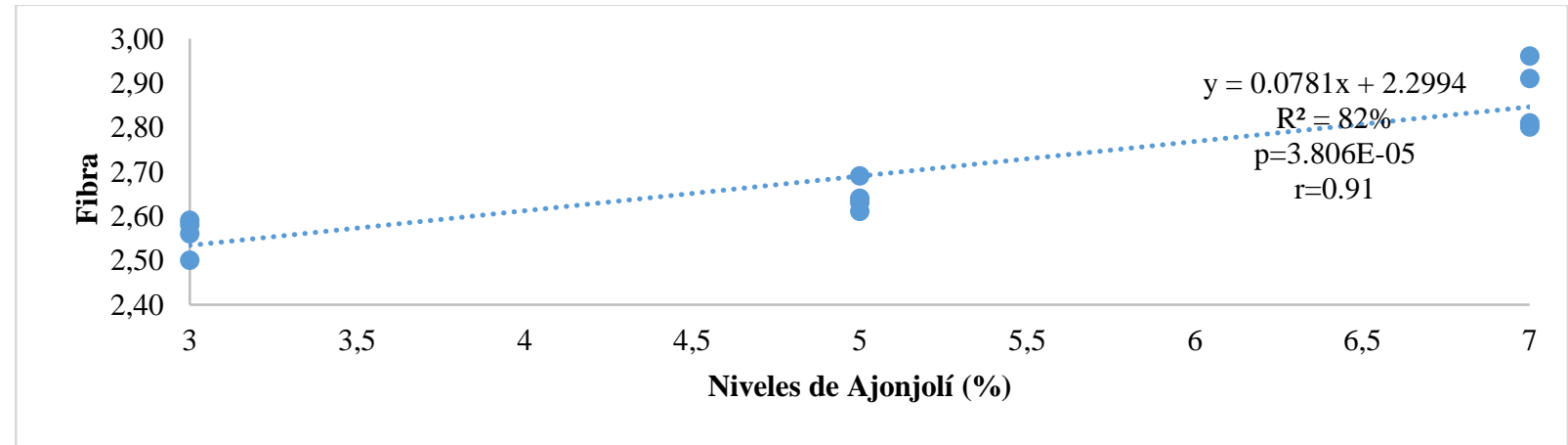

Fuente: Elaboración propia.

\section{Capacidad Antioxidante}

La capacidad antioxidante en los tratamientos difieren estadísticamente $(\mathrm{P}<0.001)$, en la Tabla 2 denotan los valores de $\mathrm{Rf}$ que se reportaron en los diferentes días de análisis. El valor promedio más alto es 1.24 corresponde al nivel $7 \%$ de ajonjolí, los niveles 3 y $5 \%$ contienen un promedio 0.53 y 0.83 de $\mathrm{Rf}$ para el recorrido de Vitamina $\mathrm{E}$, mientras que en el tratamiento con $0 \%$ de ajonjolí no determina la presencia de esta. 
ISSN: 2600-5859

Tabla 2. Identificación del valor Rf por cromatografía de capa fina del yogur tipo III con zapallo y ajonjolí en los días 1,10 y 21 .

\begin{tabular}{|c|c|c|c|c|c|c|c|c|c|c|}
\hline \multirow{2}{*}{$\begin{array}{c}\text { Variable } \\
\text { Vitamina E }\end{array}$} & \multicolumn{8}{|c|}{ Tratamientos } & \multirow{2}{*}{ E.E. } & \multirow{2}{*}{ Prob. } \\
\hline & $0^{\circ}$ & & $3 \%$ & & $5 \%$ & & 7 & & & \\
\hline Día 1 & nd & a & 0.55 & b & 0.84 & C & 1.24 & $d$ & 0.01 & $<0.0001$ \\
\hline Día 10 & nd & a & 0.52 & $\mathrm{~b}$ & 0.83 & c & 1.27 & d & 0.01 & $<0.0001$ \\
\hline Día 21 & nd & a & 0.54 & b & 0.82 & c & 1.23 & d & 0.01 & $<0.0001$ \\
\hline
\end{tabular}

Fuente: Elaboración propia.

Análisis de resultados organolépticos del yogur tipo III con zapallo y ajonjolí como aporte de fibra y antioxidantes.

En la Tabla 3, se puede ver los resultados de los parámetros organolépticos del yogur tipo III con zapallo y ajonjolí a los días 1 y 21.

Tabla 3. Características organolépticas del yogur tipo III con zapallo y ajonjolí como aporte de fibra y antioxidantes.

\begin{tabular}{|c|c|c|c|c|c|c|c|c|c|c|}
\hline \multirow{3}{*}{\begin{tabular}{|c|} 
Variable \\
\\
Color \\
Día 1
\end{tabular}} & \multicolumn{8}{|c|}{ Tratamientos } & \multirow{2}{*}{ E.E. } & \multirow{2}{*}{ Prob. } \\
\hline & \multicolumn{2}{|c|}{ O\% } & \multicolumn{2}{|c|}{$3 \%$} & \multicolumn{2}{|l|}{$5 \%$} & \multicolumn{2}{|l|}{$7 \%$} & & \\
\hline & 3.93 & $\mathbf{b}$ & 3.67 & $a b$ & 4.13 & $\mathbf{b}$ & 3.13 & a & 0.18 & 0.0018 \\
\hline Día 21 & 4.27 & $\mathbf{b}$ & 4.00 & $\mathbf{b}$ & 4.20 & $\mathbf{b}$ & 3.27 & $\mathbf{a}$ & 0.14 & $<0.0001$ \\
\hline Variable & \multicolumn{8}{|c|}{ Tratamientos } & $\mathbf{E} \mathbf{E}$ & Prob \\
\hline Olor & \multicolumn{2}{|c|}{$0 \%$} & \multicolumn{2}{|c|}{$3 \%$} & \multicolumn{2}{|l|}{$5 \%$} & \multicolumn{2}{|l|}{$7 \%$} & Eole & 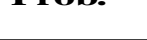 \\
\hline Día 1 & 3.53 & $\mathbf{a}$ & 3.53 & $\mathbf{a}$ & 4.07 & $\mathbf{a}$ & 3.67 & $\mathbf{a}$ & 0.19 & 0.1577 \\
\hline Día 21 & 3.87 & $\mathbf{b}$ & 3.60 & $\mathbf{a b}$ & 4.00 & b & 3.13 & $\mathbf{a}$ & 0.16 & 0,0015 \\
\hline Variable & \multicolumn{8}{|c|}{ Tratamientos } & $\mathbf{F} \mathbf{T}$ & Prob \\
\hline S abor & \multicolumn{2}{|c|}{$0 \%$} & \multicolumn{2}{|c|}{$3 \%$} & \multicolumn{2}{|l|}{$5 \%$} & \multicolumn{2}{|l|}{$7 \%$} & E.E. & PFob. \\
\hline Día 1 & 3.20 & $\mathbf{a}$ & 3.33 & $\mathbf{a}$ & 4.20 & b & 3.93 & $a b$ & 0.23 & 0.0071 \\
\hline Día 21 & 3.53 & $\mathbf{a}$ & 3.40 & $\mathbf{a}$ & 3.40 & a & 3.00 & $\mathbf{a}$ & 0.18 & 0,1853 \\
\hline Variable & \multicolumn{8}{|c|}{ Tratamientos } & $\mathbf{E} \mathbf{E}$ & Prob \\
\hline Textura & \multicolumn{2}{|c|}{$0 \%$} & \multicolumn{2}{|c|}{$3 \%$} & \multicolumn{2}{|l|}{$5 \%$} & \multicolumn{2}{|l|}{$7 \%$} & E.E. & Prob. \\
\hline Día 1 & 3.13 & $a b$ & 3.60 & $a b$ & 4.00 & b & 2.87 & $\mathbf{a}$ & 0.25 & 0.0101 \\
\hline Día 21 & 4.27 & $\mathbf{b}$ & 4.00 & $\mathbf{b}$ & 4.20 & b & 3.27 & $\mathbf{a}$ & 0.14 & $<0.0001$ \\
\hline
\end{tabular}

Fuente: Elaboración propia.

E.E $=$ Error Estándar

Prob > 0,05: no existe diferencias estadísticas

Prob < 0,05: existe diferencias significativas

Prob < 0,01: existen diferencias altamente significativas

Medias con letras iguales en la misma fila no difieren estadísticamente de acuerdo a la prueba de Tukey. 


\section{Olor (puntos)}

En la valoración del olor del yogur tipo III con zapallo y diferentes niveles de ajonjolí, las calificaciones asignadas para el día 1 no presentaron diferencias significativas $(P>0,05)$, con los niveles de ajonjolí $0,3,5$ y 7\%, en el día 21 tenemos diferencias altamente significativas (P > 0,001) dando la calificación de Me gusta en el tratamiento de 5\% de ajonjolí, mientras que los demás no gusta ni disgusta su olor.

\section{Color (puntos)}

En la valoración del color del yogur tipo III con zapallo y diferentes niveles de ajonjolí, las calificaciones asignadas presentaron diferencias significativas $(\mathrm{P}>0,001)$, con los niveles de ajonjolí 0, 3, 5 y 7\%, permitió registrar en el día 1 puntuaciones de 3.93, 3.67, 4.13 y 3.13 / 5 respectivamente, en el día 21 tenemos la valoración de 4.27, 4.00, 4.20 y 3.27 / 5, correspondiendo en el día 1 y 21 la calificación de Me gusta en los diferentes niveles de ajonjolí. Además se observa en los días evaluados que el tratamiento con $7 \%$ no gusta ni disgusta de los demás, por lo que podemos decir que a mayor nivel de ajonjolí el color difiere desde la percepción de los consumidores, los cuales asignan valores aceptables al producto como tal.

\section{Textura (puntos)}

En la valoración de la textura del yogur tipo III con zapallo y diferentes niveles de ajonjolí, las calificaciones asignadas para el día 1 presentaron diferencias significativas $(\mathrm{P}>0,05)$, con los niveles de ajonjolí $0,3,5$ y 7\% dando la valoración de 3.13, 3.60, 4.00 y 2.87 / 5 dando el tratamiento con 5\% de ajonjolí una calificación de Me gusta lo que difiere de los demás; en el día 21 encontramos diferencias altamente significativas $(\mathrm{P}<0.0001)$ con los niveles de ajonjolí 0, 3, 5 y 7\% dando la valoración de 4.27, 4.00, 4.0 y 3.27 / 5 dando una calificación de Me gusta para los 3 primeros niveles lo que difiere del nivel de $7 \%$ que no gusta ni disgusta.

\section{Sabor (puntos)}

En la valoración del sabor del yogur tipo III con zapallo y diferentes niveles de ajonjolí, las calificaciones asignadas para el día 1 presentaron diferencias altamente significativas $(\mathrm{P}>$ 0,0001), con los niveles de ajonjolí 0, 3, 5 y 7\% dando la valoración de 3.20, 3.33, 4.20 y 3.93 / 5 dando el tratamiento con 5\% de ajonjolí una calificación de Me gusta lo que difiere de los demás; en el día 21 no encontramos diferencias estadísticas $(\mathrm{P}>0,05)$.

\section{Análisis de resultados microbiologicos del yogur tipo III con zapallo y ajonjolí como aporte de fibra y antioxidantes.}


ISSN: 2600-5859

Vol. 3, N².1, p. 94-107, mayo, 2020

Los análisis microbiológicos realizados a los tratamientos de yogur elaborado con distintos niveles de ajonjoli, determinaron la ausencia de Coliformes totales, E. coli, Staphilococcos, Mohos y Levaduras, totales como se muestra en la Tabla 11-3 esto nos indica que se cumple con los requerimientos de la Norma INEN (2006), que indica que el yogur debe estar libre de microorganismos, debiendo presentar únicamente los gérmenes de la flora normal del yogur.

\section{Conclusiones.}

- La inclusión de niveles de ajonjolí en el yogur tipo III con zapallo, difieren estadísticamente $(\mathrm{P}<0.001)$ en cada tratamiento. El valor promedio más alto es $2.88 \%$ corresponde al nivel 7\% de ajonjolí, los niveles 0,3 y $5 \%$ contienen un porcentaje promedio de $2.25,2.54$ y $2.64 \%$ de fibra respectivamente, lo que indica que el aumento de niveles de ajonjolí aporta mayor cantidad de fibra al producto terminado.

- Con los niveles de ajonjolí que se añadieron en el yogurt de zapallo tipo III se evidencio que porcentaje de capacidad antioxidante en los tratamientos difieren estadísticamente $(\mathrm{P}<0.001)$, donde el valor promedio más alto es $1.24 \mathrm{mg}$ corresponde al nivel 7\% de ajonjolí, los niveles 3 y 5\% contienen un valor promedio de 0.53 y $0.83 \mathrm{mg}$ de vitamina E correspondiente a la capacidad antioxidante, mientras que en el tratamiento con inclusión de $0 \%$ de ajonjolí no se determina, por lo que se demuestra que los tratamientos con inclusión de niveles de ajonjolí presentan contenido de Vitamina E como capacidad antioxidante.

- La inclusión de niveles de ajonjolí, afectó estadísticamente a las propiedades físico químicas en cuanto a $\mathrm{pH}$, materia seca, aporte proteico y grasa en la valoración realizada a los días 1,10 y 21 días.

\section{Referencias bibliográficas.}

Bareiro, J. (2011). Hortaliza rustica y versatil. Recuperado de: http://www.Ini.unipi.it/stevia/Supplemento/PAG35009.HTM\#arriba.

Madrid y Cenzano, J. (1994). Nuevo manual de industrias alimentarias. Edición ampliada y corregida. 372. Madrid : Mundi prensa libros. págs. 78-83.

Mejía, V. (2006). Extracción del gel de Opuntia ficus para la elaboración de yogurt dietetogeriátrico. . Riobamba, Ecuador : Tesis de Grado. Facultad de Ciencias Pecuarias. ESPOCH. pag. 56-80. 
NTE INEN 2395. (2006). Leches Ferentadas, Requisitos. Norma Técnica Ecuatoriana.

Recuperado de: 2006. https://studylib.es/doc/5365063/nte-inen-2395--lechesfermentadas.-requisitos.

ORG. (2015). Yogures fermentados con mas vitamina E. Recuperado de: https://alimentos.org.es/yogures-y-leches-fermentadas-con-mas-vitamina-e.

Pacheco, D. y Emperatriz. (2007). Efecto de la acetilacion, succinilacion y la hidrolisis enzimatica sobre las propiedades funcionales de la harina de ajonjoli.

PEARSON, (D). (2007). Curcubitáceas. México : Editorial Trillas, pág. 125.

Pehanich, M. (2007). Piensa en Fibra. Recuperado de: http//wwwmundolacteoycarnico.com..

Pérez, C. (2017). Zapallo: propiedades y beneficios. Zapallo: propiedades y beneficios. Recuperado de: https://www.natursan.net/zapallo-propiedades-y-beneficios/.

Porter, J. (1981). Leche y productos lacteos. Segunda edicion . Madrid: acribia, 1981. pág. 12.

Sacón, P. (2004). Efecto de cuatro niveles de estabilizante (0.9, 1.1, 1.3 y 1.5\%) para la coagulación de yogurt persa. Riobamba, Ecuador : Tesis de Grado. Facultad de Ciencias Pecuarias. ESPOCH. Riobamba, Ecuador, pag. 41-61.

Vanguardia, L. (2018). Calabaza: propiedades, beneficios y valor nutricional. [En línea]

Vayas, E. (2002). Resumenes de la materia procesamiento de leche Octavo sementre Facultado de Ciencias Pecuarias. Riobamba: Ecuador, págs. 7 - 18. 


\section{PARA CITAR EL ARTÍCULO INDEXADO.}

Morales Caluña, A. de los Ángeles, Erazo Rodríguez, F. P., Paredes Peralta, A. V., \& Sánchez Herrera , T. E. (2020). Desarrollo de yogur tipo III con zapallo y ajonjolí como aporte de fibra y antioxidantes . ConcienciaDigital, 3(2.1), 97-107. https://doi.org/10.33262/concienciadigital.v3i2.1.1222

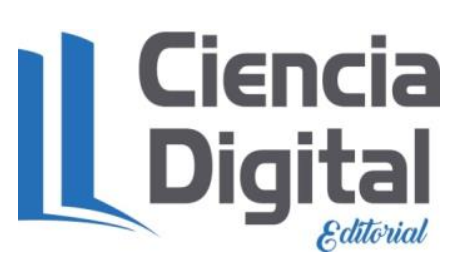

El artículo que se publica es de exclusiva responsabilidad de los autores y no necesariamente reflejan el pensamiento de la Revista Conciencia Digital.

El artículo queda en propiedad de la revista y, por tanto, su publicación parcial y/o total en otro medio tiene que ser autorizado por el director de la Revista Conciencia Digital.
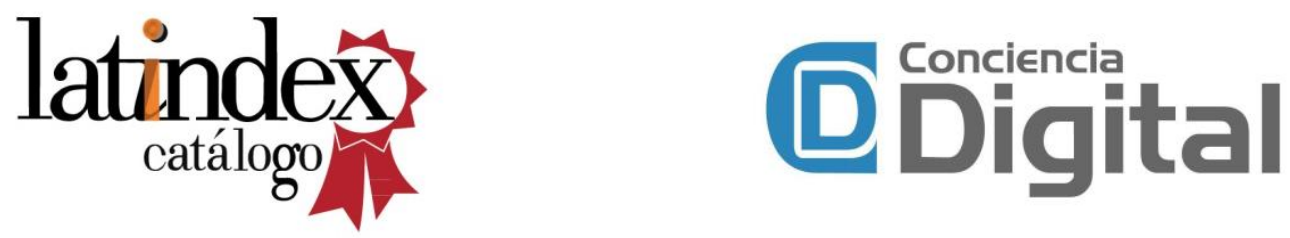